\title{
Fronteras simbólicas y clases medias. Movilidad social en Chile
}

\author{
Mayarí Castillo*
}

Perfiles Latinoamericanos, $24(48)$

2016 | pp. 213-241

DOI: $10.18504 / \mathrm{pl} 2448-009-2016$

\section{Resumen}

A más de tres décadas de la aplicación de las políticas de ajuste estructural en Chile, las clases medias han aumentado su peso en la estructura social y han cambiado su sector económico, empleo y trayectorias de movilidad social. Este artículo analiza la percepción de las fronteras simbólicas que, emergentes de estos procesos macrosociales, van moldeando las subjetividades de los sujetos pertenecientes a las clases medias en procesos de movilidad social ascendente y se relacionan con las percepciones sobre la desigualdad. Para esto se presenta un análisis cualitativo de casos, en los que destacan componentes discursivos como la meritocracia, el esfuerzo y la idea de "barreras/obstáculos" como ejes discursivos claves. Este artículo se inscribe dentro de los recientes estudios sobre clases medias para el caso chileno, enfocados en los aspectos culturales tras la reproducción de la estratificación y desigualdad en Chile.

\begin{abstract}
After more than three decades of the implementation of the policies of structural adjustment in Chile, the middle classes increased their weight in the social structure and changed their economic sector, occupation and trajectories of social mobility. This article analyzes the perception of symbolic boundaries, which emerging of these macro-processes and shapes the subjectivity of middle class subjects into upward mobility processes and modifies their perceptions of inequality. Whit this objective the paper presents a qualitative analysis of cases in which discursive components like meritocracy, the effort and the idea of "barriers/obstacles" became a key discursive axis. This article is inscribed within the recent studies on middle classes for the Chilean case, which focus specially on the cultural aspects after reproduction of stratification and inequality in Chile.
\end{abstract}

Palabras clave: clases medias, movilidad social ascendente, desigualdad, metodología cualitativa, discursos, fronteras simbólicas, Chile.

Keywords: Middle classes, upward social mobility, inequality, qualitative methodology, discourses, symbolic boundaries, Chile.

* Doctora en Sociología, Freie Universität Berlin. Académica Escuela de Antropología, Universidad Academia de Humanismo Cristiano. 


\section{Introducción ${ }^{1}$}

no de los sectores clave para la comprensión de las transformaciones de la sociedad chilena en los últimos treinta años son las clases medias. A casi más de tres décadas de la aplicación de las políticas de ajuste estructural, ese sector ha mostrado cambios relevantes en la franja económica en que se encuentran, en tipo de relación laboral y empleo, así como en sus trayectorias de movilidad social. Sobre estos tres puntos se ha agrupado buena parte de la investigación en el tema durante las últimas décadas, casi siempre desde una perspectiva cuantitativa (León \& Martínez, 2001; Cerda, 1998; Martínez \& Tironi, 1982, 1985; Portes \& Hoffmann, 2003; Barozet \& Espinoza, 2008; López, Rigolini \& Torche; López \& Juárez, 2012, 2012b; Méndez \& Gayo, 2007; Solimano, 2005). Sin embargo, y a pesar de lo que se ha avanzado en el estudio de estos sectores en el país en las últimas décadas, la investigación sobre las transformaciones identitarias que estos cambios implican recién ha adquirido alguna notoriedad (Silva, 2005; Méndez, 2009a, 2009b; Méndez \& Barozet, 2012; Stillerman, 2010b, 2010a). En esta línea, este artículo busca aportar, desde esta perspectiva, enfocándose a un análisis de la construcción simbólica de las posiciones de clase media en el marco de los procesos de movilidad social ascendente, a través de los discursos de sus protagonistas.

Los cambios en los patrones de movilidad social han sido uno de los fenómenos que han transformado más contundentemente a la sociedad chilena en las últimas décadas. Las reformas económicas aplicadas a principios de los ochenta modificaron el régimen de empleo y la orientación de la economía nacional, redujeron el sector público de manera importante y acrecentaron la importancia del sector privado en el país.

A partir de estas modificaciones, y a raíz de un importante aumento de la movilidad social ascendente y horizontal, las capas medias se volvieron más importantes y heterogéneas (Torche, 2005; Torche \& Wormald, 2007; Torche \& López-Calva, 2012). Todo esto encuentra también un importante marco de referencia en los cambios observados en los sectores en la base de la estratificación social, a partir de la década de los ochenta, en tanto que forman parte de estos procesos de movilidad y les otorgan sentido en el marco de sus biografías y de una reestructuración de su relación con la política y la sociedad en su conjunto

1 Investigación financiada por el Centro de Estudios Interculturales e Indígenas (CIIR), código de proyecto: 15110006; Proyecto PAI Conicyt no. 79130004 y FONDECYT de Iniciación 11140008. Se agradecen los comentarios de Emmanuelle Barozet a la versión preliminar del texto que dio origen a este artículo. 
(Campero, 1987; Cabalin, 2012; Portes \& Hoffman, 2003; Posner, 1999; Castillo, 2009).

Pese a la trascendencia de estos procesos de movilidad, los aspectos subjetivos involucrados han sido escasamente abordados. Como señala Méndez (2002: p. 27) —uno de los pocos estudios de carácter cualitativo que se puede encontrar sobre este tema para el caso chileno- , aunque se han incorporado a estos análisis algunas variables orientadas a medir percepciones, el interés predominante de los investigadores en los cambios en patrones generales de la movilidad, a partir de las políticas de ajuste estructural, ha dejado un espacio marginal a la comprensión de cómo estos procesos afectan la configuración sociocultural de las sociedades contemporáneas.

Sin embargo, la forma en que los procesos de movilidad social son experimentados y significados por las sociedades no es un tema menor: a nivel micro, los patrones de movilidad modifican la percepción del espacio social en los sujetos, sobre todo en el caso de sociedades altamente desiguales, como la chilena, en las que el peso diferenciado de la adscripción o el logro impacta en las expectativas de los individuos y sus biografías personales, conformando con el tiempo grupos con identidades distintas y disposiciones diferenciadas (Arellano, 2010; Sánchez, 2007).

A nivel macro, estos patrones de movilidad modifican las dinámicas del campo político, los niveles de conflicto y la configuración de actores en determinadas coyunturas en una sociedad (Londoño, 2011). Para el caso chileno en particular, Espinoza (2006) ha destacado el papel de este fenómeno en la configuración de actores políticos y señalado que las tendencias de movilidad social ascendente observadas en las últimas décadas han desencajado las posiciones históricas de los sujetos, disminuyendo su posibilidad de acción colectiva debido a que ha aumentado la distancia simbólica entre clases medias y trabajadores no calificados (Espinoza, 2006).

En una dirección similar, se ha indicado que una mayor fluidez entre posiciones en sentido ascendente parece fortalecer el ideal meritocrático y favorecer una apuesta por la acción individual para la obtención de la posición deseada (Bengoa, Márquez \& Aravena, 1999). En Chile, el aumento de la movilidad social ascendente y horizontal coincidió durante un periodo con una disminución de la protesta social, un deterioro de los indicadores de participación política y con una heterogenización de identidades sociales, por lo que se especuló acerca de una relación con este fenómeno, sobre todo para el caso de las clases medias (Balbontín, 2007a, 2007b). Sin embargo, la reciente conflictividad social y la emergencia de nuevos grupos sociales parecen apuntar más bien a la necesidad de relativizar estos supuestos y fortalecer la investigación empírica respecto de sus efectos a nivel sociocultural. 
En esta línea, resulta relevante considerar dos elementos propios de las dinámicas de movilidad social en Chile: en primer lugar, el patrón chileno muestra un aumento importante de los niveles de fluidez en las dos últimas décadas, donde el movimiento ascendente y horizontal es el más relevante, focalizado en particular en los segmentos medios (Torche, 2005; Torche \& Wormald, 2007; Torche \& López-Calva, 2012). Esto es central, pues uno de los grandes cambios socioculturales ha tenido relación con la producción discursiva de la idea de Chile como una "sociedad de clase media", orientado a destacar el carácter "nuevo" o "emergente" de estos segmentos.

En segundo lugar, las percepciones sobre movilidad social se han construido en el contexto de un crecimiento sostenido en las últimas tres décadas, que contrasta con los niveles de desigualdad observados en el país, uno de los más altos de la región. Así, el aumento de la fluidez entre posiciones no necesariamente ha afectado la distribución del ingreso en el país, que se ha mantenido relativamente estable en las últimas décadas y que ha llevado a Torche (2005) a referirse a la estructura social chilena como "desigual pero fluida".

Se advierte que el patrón de movilidad en Chile, pese al aumento de la fluidez entre posiciones, exhibe una relativa estabilidad en la distribución de recursos y niveles de desigualdad, que a la larga va delimitando grupos relativamente estables en el tiempo y con cierta homogeneidad interna. En esa línea, este artículo se orienta a documentar y analizar la percepción, construcción y reproducción de estas fronteras simbólicas que, emergentes de estos procesos macrosociales, moldean las subjetividades de los sujetos de clases medias en procesos de movilidad social.

En estos casos, los sujetos han reescrito su relato biográfico a la luz de este tránsito, por lo que a través de este análisis se busca aportar con evidencia empírica, a la discusión sobre la importancia de los aspectos subjetivos de la movilidad social, presentando el análisis de dieciocho casos de individuos clasificados como clases medias, todo ellos residentes en la región metropolitana.

En este artículo se toma como base teórica para la definición de la idea de clases el trabajo de Pierre Bourdieu (2007, 2000), en tanto este permite entender la estrecha relación entre la estructura de relaciones objetivas delimitada a partir de la distribución de los distintos tipos de capital y la producción simbólica imbricada en el proceso de constitución y construcción de una clase social. En su texto clásico, "Cómo se hace una clase social", Bourdieu (2000) refiere al estrecho vínculo entre ambas dimensiones y menciona el rol sumamente relevante que la dimensión simbólica tiene en el caso de las posiciones intermedias, dada su mayor heterogeneidad y carácter contradictorio. Pese a que Bourdieu no desarrolló trabajo específico bajo la noción de clases medias o posiciones intermedias, la fuerte preocupación del autor 
sobre las nociones de clase y estatus, producto de la influencia de los autores clásicos sobre su pensamiento, lo llevaron a conducir investigaciones empíricas en las cuales el papel de los sectores intermedios, su constitución simbólica, sus trayectorias de movilidad y su carácter contradictorio está presente, como es el caso de La distinción y Los herederos (Bourdieu, 1979; Bourdieu \& Passeron, 2003).

Es precisamente a partir del carácter contradictorio de las posiciones de clases medias el punto central que utiliza Erik O. Wright (2009) para su trabajo orientado a la reelaboración de la tradición de los estudios de estratificación, tributarios del concepto marxista de clase social. Intentando resolver uno de las principales falencias de este concepto en el marco de la tradición marxista - la ausencia de reflexión sobre los grupos intermedios o clases medias-, Wright desarrolla un esquema que recoge la influencia weberiana y que se orienta a la delimitación de posiciones de clase a partir de elementos ubicados fuera de los esquemas clásicos del marxismo, como la organización, autonomía laboral y los niveles de formación, sumamente relevantes en las sociedades contemporáneas y presentes también en el pensamiento de Bourdieu.

Así, Wright desarrolla un esquema basado en la distribución desigual de tres tipos de capital: de producción, de organización y de cualificación, a partir de cuya combinatoria genera doce grupos teóricos que permiten visibilizar a las clases medias, situándolos en los pequeños empleadores y la pequeña burguesía en el polo de los propietarios y en los directivos titulados, directivos no titulados, supervisores expertos y no directivos expertos, en el polo de los no propietarios. A partir de este esquema, el autor muestra la diversidad de las clases medias y su carácter contradictorio, entregando así herramientas específicas para un análisis desde el concepto de clase social enfocado a estos segmentos.

Por tal razón, para este artículo se recupera el trabajo de estos autores. Con el fin de generar una definición para la selección de una muestra teórica de casos, se consideraron cuatro grupos dentro de las clases medias: pequeños propietarios, directivos no profesionales, técnicos en cargos de responsabilidad — supervisión (con al menos una persona a su cargo)—, y profesionales autónomos o asalariados. Para la selección de la muestra, se cuidó la inclusión de sujetos de cada uno de estos grupos ocupacionales. En tanto que para dar cabida a la dimensión de consumo y estilo de vida - tan relevante en el trabajo de Bourdieu sobre producción simbólica de clases sociales-, se introdujo la variable ingreso en los criterios de selección de casos, ya que a pesar de compartir un determinado tipo de capital, de calificación y de control sobre el proceso laboral, las diferencias en términos de ingreso implican diferencias sustantivas entre las clases medias. Se generaron así tres grupos de ingreso a 
considerar en la selección de los entrevistados. ${ }^{2}$ Finalmente, en atención a la complejidad del tratamiento del fenómeno de movilidad social en los estudios de estratificación, se buscó introducir criterios de selección de casos que permitieran, desde un punto de vista operativo, distinguir sujetos involucrados en procesos de movilidad social intergeneracional.

Así, se seleccionaron entrevistados que proviniesen de hogares cuyo jefe de hogar tuviera una ocupación principal de clase trabajadora, la cual fue delimitada a partir de los grandes grupos del código ciuo. ${ }^{3}$ Mediante la combinatoria de estos atributos, se conformó una muestra de dieciocho casos, diez mujeres y ocho hombres, a quienes se aplicaron entrevistas en profundidad sobre percepciones de estratificación social, desigualdad y participación política.

Este trabajo se basa en el análisis del material cualitativo agrupado en torno al primer eje temático de las entrevistas y fue llevado a cabo mediante el software Nvivo, herramienta orientada a la sistematización y generación de esquemas complejos de análisis cualitativo en una segunda etapa de trabajo. Para el análisis con dicho software, se utilizó la teoría fundamentada de Glaser (1998) con la que se generaron categorías teóricas de alcance intermedio que permitieran el análisis de los procesos de movilidad social en términos subjetivos. Se realizó primero una codificación libre y luego, en una siguiente etapa, se agruparon las principales categorías emergentes, para establecer las principales dimensiones o subcategorías a considerar en los elementos teóricos que debían elaborarse en una tercera etapa.

En este tercer momento, se agruparon las categorías de abstracción baja en las categorías teóricas principales expuestas en este artículo como herramientas interpretativas: el movimiento ascendente, la noción de mandato y las barreras. El resultado final del proceso se presenta en calidad de esquema teórico de alcance intermedio, susceptible de ser aplicado en casos similares, previo ajuste y revisión de pertinencia.

2 Se introdujo la variable ingreso a partir de lo estimado por GSE de los grupos C2 (ingreso promedio por hogar de 1073.000 pesos chilenos-2217 usD) y C3 (ingreso promedio por hogar de 517.000 pesos chilenos-1068 usD). Sin embargo, y dada la definición de clase media utilizada, sectores limítrofes del segmento ABC1 y D también debían ser considerados, en función del tipo de empleo, ańos de estudio y patrimonio. Dado que el promedio de ingreso de un hogar $\mathrm{ABC} 1$ asciende a los 2.866 .000 pesos chilenos-5924 usD y de un hogar perteneciente al segmento D a 292.00 pesos chilenos-603 usD, se estableció un límite inferior de 350000 pesos chilenos-723.469 usD por hogar y de 3000000 pesos chilenos-6201 usD en el caso del límite inferior. Con base en esto, se establecieron tres intervalos para la selección de entrevistados: ingreso alto (723.469-1033 USD); ingreso medio (1035-2067 usD) e ingreso bajo (2069-6201 usD).

3 Las ocupaciones incluidas en los grupos 5 (trabajadores de los servicios y vendedores de comercios y mercados), 6 (grupo 6: agricultores y trabajadores calificados agropecuarios y pesqueros), 7 (oficiales, operarios y artesanos de artes mecánicas y de otros oficios (calificados), 8 (operadores de instalaciones y máquinas y montadores (calificados/semicalificados) y 9 (trabajadores no calificados), fueron calificados como clase trabajadora. 


\begin{abstract}
Me gustaba que se llamase Yasna, me gustan los nombres que avisan la pobreza, esos nombres como Jhonatan y Jenifer, esos nombres que dicen cuidado con el perro quiltro, que marcan terreno, que avisan la selva. La clase media hace chiste con

esos nombres, les parecen ridículos y graciosos sobre todo cuando son deformaciones anglo que se mezclan con un pobre Pérez o un pobre Carrasco, se ríen y en la noche se van a la fiesta kitsch de La Blondie. Se llamaba Yasna y la amé tanto.

La clase media se ríe en función de los nombres connotadores de pobreza, ahí intentan marcar su diferencia, sus privilegios, su idílico origen común. Pero la clase media omite los apellidos, ellos también son Martínez y Hernández, aunque antecedidos por Camilos, Ignacios y Franciscas.

Pablo Paredes, Cuidado con el perro quiltro
\end{abstract}

Todo proceso de movilidad social es relativamente doloroso en términos subjetivos y los casos analizados no son la excepción. Todos vienen de hogares susceptibles de ser clasificados como "clase trabajadora", buena parte de estos sujetos han sido marcados por experiencias de vulnerabilidad y discriminación $y$, si bien todos residen en la región metropolitana, muchos de ellos tienen trayectorias familiares vinculadas a las dinámicas de migración campo-ciudad prevalentes desde los años sesenta en Chile.

En el marco del proceso de movilidad social en el que se ven envueltos, estos sujetos han debido abandonar un "lugar" que les resulta familiar y acostumbrarse a otro, seleccionando en el camino lo que deben conservar y lo que es preciso adquirir para legitimar su nueva posición. En términos de Bourdieu (2000), estos sujetos han tenido que modificar sustancialmente su habitus mediante la adquisición de nuevos elementos que les han permitido desenvolverse en su nuevo espacio y — pese a la dificultad que esto implica — no se observan casos de habitus desgarrados, sino más bien reconfiguraciones reflexivas a partir de las experiencias cotidianas. Lejos de una adaptación preconsciente, se asiste a un proceso en el que el sujeto se torna creativo y flexible en el momento en que se enfrenta a la situación y reflexivo al momento de evaluarla a largo plazo. Las articulaciones identitarias (Hall, 2000) de estas clases medias se van modificando y adaptando contextualmente y los elementos que constituyen su discurso adquieren peso diferenciado, dependiendo de quiénes son los interlocutores, 
quiénes son los pares y las características del espacio donde se está actuando. Un ejemplo de esto se observa a continuación:

Yo era bien matea ${ }^{4}$ cuando chica, pero me sentía mal en mi nuevo colegio. Yo las veía como más cuicas, ${ }^{5}$ esa era mi percepción, que habían tenido otro colegio, quizás de niñas, que siempre habían estudiado en colegios como de monjas y yo venía de un colegio súper rasca, ${ }^{6}$ sentí que las niñas eran distintas y nada que ver, me sentía más pobre. A mi mamá le pasó lo mismo en las reuniones de padres y también se adaptó. Yo también me adapté. Mis mejores amigas son de ahí ahora y me di cuenta que yo las veía distinto de lo que eran en verdad. (Profesional directivo en cargo de responsabilidad, 32 años, ingreso alto).

Así, todo proceso de movilidad —ascendente, descendente u horizontalimplica pérdida de redes y conocimiento práctico si se evalúa el plano de lo negativo. Implica también la generación de nuevas formas de sociabilidad y adquisición de conocimientos, si se observa el plano de lo positivo. En el caso de la movilidad ascendente, este proceso no es menos difícil, ya que los individuos deben situarse en un espacio que sienten como ajeno y validarse ante otros (Svampa, 2003), a la vez que deben reelaborar la narrativa sobre su propia trayectoria a la luz de este nuevo habitus.

En todos los casos analizados, los individuos han lidiado con lo que perciben como desventajas derivadas de su origen social, generalmente identificadas con los diferenciales de capital cultural, social y económico. También han debido lidiar con una serie de dinámicas de diferenciación que, inscritas en el orden de lo simbólico, establecen las distancias sociales entre sujetos que coexisten en el mismo espacio y remarcan las diferencias entre ellos de manera constante.

Los relatos analizados muestran particularidades en la forma en que este proceso ha sido incorporado en la narrativa biográfica: en algunos casos, la sensación de haberse "salvado" de la "mala pata colectiva" que supone haber nacido en un contexto empobrecido — parafraseando a Bourdieu (1999) — es procesada de tal manera que la creencia meritocrática se constituye en uno de los principales componentes de la articulación identitaria de clase media. En otros casos, la percepción de permanente desventaja se sitúa al centro de la construcción y fortalece la percepción de desigualdad.

\footnotetext{
Coloquial: estudiosa.

Coloquial: de clase alta.

Coloquial: pobre.
} 


\section{Construyendo significados para una movilidad ascendente}

Los discursos analizados otorgan un lugar central al proceso de movilidad en la construcción de su percepción del espacio social. A tal proceso se le relata a partir de sus significados en términos biográficos, ilustrándolo como una línea que va de menos a más, cuyo punto de inicio se encuentra en la infancia del hablante y cuya consolidación se proyecta en la generación siguiente. Los sujetos se sitúan como punto crítico de partida y punta de lanza de la movilidad del grupo familiar, entendiendo que este incluye no solo la familia conformada a partir de su adultez, sino también a los miembros del grupo familiar del hogar de origen. En ese marco, la movilidad ascendente es vista como un proceso que paso a paso empuja no solo la propia trayectoria, sino también la de la familia y pares de origen.

A pesar de la heterogeneidad de los sujetos entrevistados, es posible distinguir una estructura común en los discursos sobre la movilidad, la cual se compone de tres categorías teóricas que han de desarrollarse en este artículo (el movimiento ascendente, el mandato y las barreras) y de tres tipos de interlocutores (los impulsores, los obstaculizadores y los pares legitimadores). Pero en ese tronco común también se observan variaciones que provienen de las características que delimitan la heterogeneidad de la clase media en términos de ocupación, ingreso y consolidación de la posición, y que se focalizan en la importancia otorgada a los objetivos y el bienestar individual durante el proceso de movilidad. Esas variaciones se relacionan, a su vez, con los niveles de vulnerabilidad de las distintas posiciones de clase agrupadas bajo la noción de clase media, en la que existen percepciones distintas respecto de la distribución de la vulnerabilidad: la clase media de ingreso medio y la clase media de ingreso bajo son las que perciben un mayor riesgo.

Cabe señalar que, en el contexto de un relato sobre la movilidad vinculada a las trayectorias familiares, surge la noción de mandato como una de las principales categorías extraídas del análisis. Dicha noción — la cual se revisa extensamente más adelante- articula el discurso biográfico de los entrevistados a partir de la permanente interlocución con la familia y pares de infancia, lo que condensa así todos los elementos surgidos en el análisis que refieren a las demandas que el sujeto considera que se dirigen a él durante la obtención y consolidación de la posición de clase media.

A través del mandato, el hogar de origen se convierte en un acreedor imaginario del "éxito" del individuo: este compromiso implica para los entrevistados la obligación moral de contribuir al mejoramiento de la calidad de vida del hogar de origen y de impulsar — con el ejemplo o de maneras más directas, como el 
apoyo monetario o educacional — a quienes se encuentran en la consolidación de un proyecto similar.

La centralidad del mandato en la construcción de los significados de la movilidad social hace imprescindible la inclusión del rol de la familia y de los pares de origen como interlocutores en este proceso, como señala Bertaux (1997). La evidencia recopilada en esta investigación muestra que estos significados toman forma y significado a partir de esta interlocución permanente, estructurando relaciones que incluyen dinámicas de motivación, obstaculización o retribución, para citar las más relevantes. Estos y otros elementos se analizan con detalle en los siguientes apartados.

"Salir" de la pobreza: el movimiento ascendente como dirección de la movilidad social

La primera categoría extraída del análisis alude a la percepción de la trayectoria del sujeto en el espacio social en forma de una línea que va de menos a más, que sitúa la clase baja en el polo inferior y a la clase alta en el polo superior. En este movimiento ascendente, los entrevistados comparten una visión negativa de la clase baja, realizando un ejercicio constante dirigido a establecer una diferencia del hogar de origen respecto del contexto de clase baja en el que se inserta.

En este elemento es central recordar que los sujetos provienen de espacios que se han transformado de manera importante en las últimas décadas (Portes \& Hoffman, 2003), transformaciones que han dado lugar a espacios organizacionalmente atomizados y, en muchos casos, estigmatizados. Tal estigmatización permea de manera profunda la mirada de los entrevistados, como se advierte en las citas siguientes:

Yo estudié en un colegio que era muy, muy peligroso, donde asaltaban y todo eso, con todo lo que pasaba en los colegios de allá de La Florida, en algunos como más marcados, como más estigmatizados. Los chicos me decían: "Yo no tengo para la micro, ${ }^{7}$ no tengo para almorzar hoy día. Tengo que juntar las monedas con mi mamá," cosa que yo también viví cuando chico. Pero tú no ves que mis compañeros estaban interesados en salir de eso y no me refiero a salir de eso en tener más plata, sino en tener un nivel de conocimientos mayor frente a la vida, al mundo. (Profesional asalariado, 33 años, ingreso medio).

\footnotetext{
7 Coloquial: transporte público.
} 
El pobre quiere seguir siendo pobre, yo creo que por ahí va la cosa. Porque si tú miras a un pobre, el pobre tiene todo lo de una persona que trabaja, porque tiene salud gratis, tiene beneficios gratis, tiene lucas ${ }^{8}$ que le dan con bonos, entonces realmente se le da la vida fácil y ellos no tienen como la capacidad de decir que el camino son los estudios, entonces dejan que el hijo haga lo que quiera, total después todo se lo van a dar y va a ser fácil para él todo. (Trabajador administrativo y de servicios en cargo de responsabilidad, 38 años, ingreso alto).

Por esta razón, el primer elemento discursivo contenido en el concepto de movimiento ascendente es la diferenciación del hogar de origen respecto al contexto de clase baja, lo que se realiza a partir de la identificación retrospectiva de tres fenómenos: el fomento de la educaciónlel acceso a cultura, una ética del trabajo y el deseo de superación.

Todos los conceptos anteriores se entrelazan en una narrativa que relata cómo los padres o adultos cercanos establecieron, pese a sus escasas herramientas, la importancia de estudiar en el hogar, marcando la finalización de los estudios como uno de los objetivos mínimos a alcanzar por los sujetos. Acá se alude también a la presencia de un fuerte discurso normativo acerca del valor del trabajo y de la necesidad de "salir adelante", pese a todas las dificultades:

Yo tuve la suerte de que, si bien vengo de una familia muy pobre, es una familia que leía mucho, entonces tuve mucho acceso a libros cuando chica, libros de mierda de repente, pero en mi casa había libros y estaba la importancia de estudiar, la importancia de la lectura, porque tengo vecinos que en la misma situación, un papá mecánico y la mamá con un negocio en la casa, son tipos con arresto domiciliario por microtráfico de cocaína. (Profesional asalariado, 33 años, ingreso medio).

Estos tres elementos que el individuo identifica como diferenciados en el hogar de origen, constituyen ejes clave alrededor de los cuales los sujetos articulan su pertenencia a las clases medias, adquiriendo un peso diferenciado en relación con la heterogeneidad en términos ocupacionales: quienes se ubican en el grupo de los pequeños propietarios y directivos no profesionales enfatizan el componente "deseo de superación" por sobre los otros componentes; mientras que los profesionales recalcan la importancia del "fomento a la educación” como prioritario. Estas mismas diferencias se observan en el grupo de los trabajadores administrativos y servicios calificados, para quienes el componente de "ética del trabajo" adquiere una mayor centralidad. 
El segundo elemento discursivo contenido en el concepto de movimiento ascendente es la proyección intergeneracional: se considera que el punto máximo de esta trayectoria no es posible que lo alcance el sujeto que la inicia, depositando en la siguiente generación la responsabilidad de consolidarla.

El objetivo definido por los sujetos, en el marco de esta trayectoria, no es llegar a formar parte de la clase alta, sino integrarse a lo que perciben como clase media "pudiente", que se encuentra en una posición menos vulnerable que la identificada como propia.

Esta proyección intergeneracional articula tres elementos discursivos: la aspiración sobre el futuro de los hijoslla ausencia de privaciones en la crianzalel mantenimiento de la formación ética del hogar de origen. Estos elementos estructuran un relato en torno al deseo manifestado, en todos los casos, de que los hijos sean, al menos, profesionales destacados: "Yo quiero que estudie, porque yo sé que los estudios por lo menos te da un título, no importa que no tenga pega, pero tiene un título y eso le abre más puertas" (Trabajadores administrativos y servicios calificados en cargo de responsabilidad, hombre, 38 años, ingreso bajo).

Esta importancia de la educación en las estrategias para la consolidación de las clases medias no es para nada nueva en términos históricos y no se ha modificado de manera sustantiva en las últimas décadas. Como se ha señalado en los estudios para el caso argentino, una de las principales estrategias de consolidación de las posiciones de clase media hoy sigue siendo una alta inversión en educación para los hijos. Para el caso chileno, pese a que la educación va poco a poco perdiendo fuerza como herramienta de movilidad (Espinoza, 2006), las clases medias siguen apostando por la obtención de credenciales educativas para sus hijos, lo que se manifiesta en que buena parte del ingreso familiar se destina a este ítem, sobre todo considerando la estructura del sistema educacional en Chile, en el que la educación pública muchas veces no es una opción deseable para este segmento. La justificación de este gasto no solo radica en que sus hijos accedan en el largo plazo a una profesión mejor que la que ellos tienen, sino también en que accedan a un capital cultural al que ellos no tuvieron acceso y a un círculo social que facilitará su consolidación como sujetos de clase media.

En ese sentido, si bien la educación ha perdido fuerza como herramienta de movilidad en el marco de un proceso de "salida" de la pobreza en el caso chileno, se identifica como una de las estrategias fundamentales para la consolidación de esta "salida" ya realizada en la generación anterior, y como una estrategia protectora ante una posible "caída".

Otra idea asociada a esta proyección intergeneracional es la idea de la ausencia de privaciones en la crianza. Este es uno de los elementos más polémicos del discurso, pues se liga al "que no les falte nada" a los hijos y se vincula directamente con patrones de consumo que, como se ha recalcado en los estudios 
sobre el tema para el caso chileno, es uno de los espacios claves en los que se consolidan las fronteras simbólicas dentro de los distintos segmentos de las clases medias (Ariztía, 2009; Stillerman, 2010a, 2010b). En estos estudios se destaca la importancia de las diferencias en el volumen, dirección y espacios de consumo para cada segmento de clase media, lo que es particularmente importante para el análisis de las clases medias "emergentes", para las cuales el consumo representa una herramienta fundamental de diferenciación.

En esta línea, los sujetos entrevistados establecen modelos de parentalidad que muchas veces asocian la carencia material y desprotección/negligencia, que se remite a su propia experiencia de infancia, que en muchos casos les lleva a un consumo superior a sus posibilidades en relación con los hijos: se establece como necesario que los hijos no conozcan los rigores y carencias de su infancia; asimismo, se busca restablecer las cosas "como deberían ser", a modo de relectura de la propia narrativa biográfica. Esto resulta sumamente contradictorio como guía para la acción en relación con los hijos, pues entra en conflicto con lo que ellos mismos han identificado como central en su propia narrativa biográfica: el esfuerzo y el trabajo.

Cuando era chico, yo llegué a cuidar mi casa y cuidar mi hermana y cuando era nińo no tenía muchos amigos, porque tenía que llevar a mi hermana chica al colegio, tenía que hacer el aseo, llegaba del colegio y tenía quehacer [...]. Mi viejo, como trabajaba tanto, no me pescaba mucho, nunca me llevó a un parque a jugar a la pelota. Yo tengo pocos recuerdos de que jugara conmigo. Me gustaría que mi hijo sea inquieto, que tenga hartas inquietudes, que pruebe hartas cosas, que si quiere ser deportista que pruebe, que si le gusta la lectura que lea harto. Que pueda hacer todas esas cosas que yo no pude. (Directivo no profesional, hombre, 36 ańos, ingreso alto).

En el marco de la idea de "que no les falte nada", se da una permanente reestructuración de los límites: si se les compra un juguete, se les impone una tarea; se les compra algo que "todos los compañeros de clase tienen", pero solo como forma de protección, una estrategia protectora frente a las dinámicas de discriminación, basada en la proyección de la experiencia de los individuos que se han visto expuestos a estas durante el transcurso de su proceso de movilidad.

Finalmente, el tercer elemento contenido en esta proyección intergeneracional refiere al deseo de que los hijos no pierdan lo distintivo en la formación de sus padres: mantener la formación ética del hogar de origen:

Me gustaría darle las oportunidades que yo no tuve, eso sí con esfuerzo. No ser una mamá de "te lo doy todo" porque tiene que costar, o sea "yo te lo doy, pero 
tú también [...]". Una vez me dijeron un dicho muy sabio. Una persona mayor que yo, me dijo: "Ayúdate, que yo te ayudaré. Si tú te ayudas, yo también te voy a ayudar”, entonces creo que hay que ser así, estar ahí presente, pero también hacer que se esfuercen más, que trabajen más, que también si quieren salir, carretear, que se lo costeen. (Pequeño empresario, mujer, 30 años, ingreso medio).

A pesar de los esfuerzos de los entrevistados, ellos reconocen que deben enfrentar cotidianamente la dificultad de mantener esta formación ética, en un contexto en el que no encuentran un terreno factible para su aplicación y deben observar cómo esta se va perdiendo en sus hijos:

Él me ve a mí como el que le puedo dar todo económicamente. Pide muchas cosas, entonces de cien cosas que me pide, yo le digo 98 cosas que no. El otro día conversé con él y me dijo que yo soy un papá muy clásico, porque no lo dejo desarrollarse. "¿Pero por qué?. —Sí, porque yo quiero jugar tenis y tú no me dejas". Le pagué una escuela de kárate, le pagué una escuela de fútbol y no le puedo pagar una escuela de tenis porque yo trabajo y no puedo acompañarle". (Directivo no profesional, hombre, 36 años, ingreso alto).

\section{"Ser más que tus padres". El mandato de la movilidad social}

La importancia otorgada al hogar de origen en los aspectos subjetivos de la movilidad social aparece con fuerza en el segundo de los elementos comunes que componen la estructura discursiva, la idea de mandato. Este refiere a que el individuo se percibe como depositario de un proyecto cuyos objetivos le son impuestos a corta edad por un otro externo, ya sean los padres, tíos o abuelos. Las narrativas tienden a encajar reiteradamente el proyecto individual en uno de carácter familiar, en el cual la trayectoria individual se constituye en una estrategia orientada a generar mejores condiciones de vida para la familia en su totalidad. En ese marco, la movilidad es un proyecto a largo plazo que se proyecta a nivel intergeneracional, asumido a una edad muy temprana y rara vez cuestionado en la etapa de madurez. Esto tiene implícita la exigencia de responder a las expectativas del contexto en el que se insertan.

Este concepto de mandato involucra la necesidad de devolver o retribuir el apoyo recibido a partir del hogar de origen: contiene un componente de retribución/deuda, que representa, en la mayoría de los casos, la prioridad de los individuos en cuanto acceden a una posición de clase media, constituyéndose en el primer proyecto personal que emprenden en cuanto tienen la libertad suficiente para hacerlo. 
Este elemento es notable si se quiere cuestionar las teorías que han recalcado el énfasis en el carácter individual de la movilidad en Chile en las últimas décadas, en desmedro de un modelo más colectivo, característico de la movilidad de la clase media en el periodo del desarrollismo (Bengoa et al., 1999). Así, mientras que la literatura sobre el tema ha destacado el peso de los procesos de individualización en la forma de afrontar y significar la movilidad social, la evidencia recabada en esta investigación matiza tal fenómeno, mostrando cómo los individuos intentan conciliar el imperativo de éxito individual con elementos más colectivos, marcados por la preservación del hogar de origen como interlocutor y espacio referencial permanente.

Esta idea de retribución/deuda presenta expresiones concretas. La primera y más evidente es la económica: los entrevistados buscan traspasar parte de su patrimonio a su hogar de origen en cuanto les es posible, incrementando su aporte, a medida que aumenta su ingreso y se consolida su posición. Este traspaso de recursos busca compartir parte de los beneficios del "éxito" de los entrevistados con quienes identifican como impulsores de la movilidad y artífices relativos de la actual posición del sujeto. En ese marco, se financian bienes de consumo, viajes, invitaciones a cenar y otras actividades recreativas:

Siempre les di, desde que empecé a trabajar, obviamente mientras más iba ganando, más les iba dando y cuando me fui mi mamá me dijo: "No, nos des más porque ahora tú vas a tener tus gastos, tu casa”, pero yo no quise, me las arreglé para poder hacer las dos cosas, porque la verdad es que ellos están acostumbrados a que mi papá gana 170 mil pesos, y mi mamá debe ganar como lo mismo, porque mi mamá tiene talleres de aeróbica por el barrio y gana por hora, entonces no es un pega que pueda $[\ldots]$ entre los dos no gastan mucho, pero a uno como hija les gusta que estén bien, que si quieren salir a comer algo rico se lo coman". (Directivo profesional, mujer, 33, ingreso alto).

Una segunda dimensión discursiva de esta retribución/deuda se vincula con la reducción de la vulnerabilidad objetiva del hogar de origen. En la medida de sus posibilidades, los entrevistados relatan acciones suyas con ese objetivo, las más comunes son la compra de inmuebles en barrios más seguros, la adquisición de mejores seguros médicos o su apoyo para el retiro de los miembros del hogar de trabajos asociados al desgaste físico, el peligro o los bajos sueldos.

Estas acciones que reducen la vulnerabilidad implican la adopción de un rol parental hacia el hogar de origen, lo cual se relaciona con una tercera forma de expresión de la retribución/deuda: la protección frente a la vulnerabilidad subjetiva del hogar de origen. Esta se refiere a todas las acciones que los entrevistados señalan como destinadas a proteger el hogar de origen de su ex- 
posición a las dinámicas de discriminación, abuso y privación que ha debido experimentar el sujeto a lo largo de su proceso de movilidad social. En función de esta protección, los individuos asumen un papel activo en la toma de decisiones del hogar de origen y se constituyen en su interlocutor en situaciones identificadas por el sujeto como difíciles de afrontar: adquisición de bienes, reclamos, quejas o trámites, situaciones médicas o legales de complejidad. Esto tiene que ver con un traspaso del conocimiento que el individuo ha adquirido durante el proceso de movilidad hacia el hogar de origen —el "saber manejarse”-, en función de la protección de este y sus miembros. Dicho traspaso no se produce solo por la acción unilateral del sujeto, sino también por la constante interpelación del hogar de origen.

Por otro lado, la idea de mandato encuentra una connotación negativa en el discurso de los sujetos: la permanente presión a la que se sienten sometidos. Por parte de los sujetos, existe una continua evaluación del cumplimiento del mandato del hogar de origen, lo cual se relaciona directamente con los niveles de satisfacción personal expresados: si bien la evaluación retrospectiva arroja un balance positivo, en varios de los casos se alude a un esfuerzo insuficiente o a la falta de ciertas metas consideradas clave, como la obtención del título universitario. Cuando dichas expectativas no se han cumplido, la idea de "haberle fallado" al hogar de origen es una fuente de gran frustración para los individuos y sitúa el imperativo del logro de estas metas como una tarea urgente en el corto plazo. Ello deriva en que, para los entrevistados, la posibilidad de pensar en la realización de un proyecto individual libremente se inaugura con el cumplimiento del mandato:

Espero en diez años más haber ido a Europa, pero me interesa ir, conocer, conocer y ojalá en diez años más habiéndole pagado un viaje a mis papás. Creo que no se han tomado vacaciones nunca en la vida más que tres días al año. Eso son como los proyectos que tengo ya a más corto plazo, y lo de Europa, ojalá el otro ańo, ojalá en invierno y después pagarles un viaje a mis papás, y después de eso como olvidarlos un poco, o sea no olvidarlos, pero ya como decir: "Ya cumplí, ya se fueron de vacaciones a una parte que ellos quieran". (Profesional asalariado, 34 años, mujer, ingreso medio).

"Ser como ellos"/"No ser como ellos". Las barreras y la discriminación en los procesos de movilidad social

El tercer eje conceptual emergente del análisis del discurso de los sujetos es el de barrera, que remonta al pasado del sujeto y constituye un recurso narrativo 
destinado a significar el proceso de movilidad como una hazaña, enfatizando y recalcando los elementos adversos que el sujeto debió sortear para que esta se produjera. Tal concepto pretende matizar el peso de la noción de mandato sobre la narrativa de movilidad, relevando el rol de la agencia individual en la dirección y velocidad del proceso de movilidad social, lo que se expresa en dos formas: 1) mostrando las diferencias con sujetos insertos durante su infancia en situaciones similares, y 2) a través de un relato detallado de los obstáculos impuestos en el pasado a la movilidad:

Yo sé lo que es pasar hambre, yo lo sé, he visto a mi mamá llorar porque no tienes para comer. O que te van a cortar la luz, el agua. Una vez casi se nos incendió la casa por estar colgados ${ }^{9}$ y después me fui a vivir sola por primera vez. Ahí con mi amiga no teníamos plata ni para calentarnos, ni para comer, ni para la micro. Un día no pudimos ni ir a trabajar porque no teníamos ni para comer, ni para la micro, ${ }^{10}$ nadie fió, nos fuimos a vivir a Puente Alto, del 36 para dentro a una villa, nadie nos dio un pan. O sea ¡nos cagamos de hambre! $Y$ no le puedes decir a tu jefe que estás cagada de hambre. (Pequeńa empresaria, 34 años, mujer, ingreso alto).

Yo siempre dije: el que quiere ser pobre, va a ser pobre toda su vida. Si yo lo digo es porque yo crecí, tuve una infancia bien especial. Éramos cinco hermanos, mi padre solamente trabajaba, no tuve una infancia de grandes lujos, todo lo contrario, entonces va en ti en cierta medida el querer doblegarle la mano al destino y decir: "Yo no quiero eso para mí, yo no quiero ser pobre. ¿̇Y qué hago? Me supero, ¿̇ cómo? Consigo trabajo". Al menos yo lo hice así, trabajé, me dediqué dos o tres años a trabajar y traté de estudiar y no se me cayó el pelo, no me morí, entonces, creo que también va en la persona, en el espíritu y lo que tú quieras tener. (Pequeño empresario, 35 años, ingreso medio).

En el pasado reciente y en el presente, esta categoría se relaciona con la exposición de los entrevistados a las fronteras entre grupos sociales y las dinámicas de discriminación, rechazo y aceptación, las que hacen de la movilidad ascendente un proceso en el que los entrevistados deben abandonar parte de lo que les constituye para adoptar los elementos que los configuran como una persona "de clase media".

Esto se realiza a partir del discurso estructurado en dos polos en tensión: vergüenza del origen/orgullo del origen, cuyo mayor o menor peso orienta la acción de los individuos en cada situación contingente. El primero se asocia a la ver-

9 Coloquial: conectarse ilegalmente a las redes de tendido eléctrico.

10 Coloquial: bus de transporte público. 
güenza y timidez narrada por los entrevistados durante los primeros contactos que marcan la salida del hogar de origen, hacia espacios de clase media y que acompañan al sujeto durante su experiencia de movilidad. En este elemento se han condensado todas las experiencias asociadas a la exposición de su origen en circunstancias o maneras que les resultan incómodas y que les impulsan a modificar ciertos aspectos de su acción cotidiana: lo inadecuado de su forma de hablar en determinados grupos o momentos, su forma de vestir, su falta de conocimiento o capacidad de desenvolverse, la conciencia de sus propias marcas corporales:

No sé por qué me junté con compañeras pitucas, ${ }^{11}$ no sé por qué, como que caí en ese grupo. Llegó otra compañera de curso, provinciana, en el primer día de clases: "Oye, ¿̇y tú de dónde eres? De Temuco, ¿y tú? De Talca". Es una gran amiga hasta ahora, pero es una chica acomodada. Después me hice amiga de otra chica también de plata y todas como con casas bonitas, ellas muy bonitas, con caras bonitas, con un pasado de colegio, no de liceo, con viajes y yo nada. Yo tratando de pasar piola $^{12}$ y dándome cuenta de "¡dónde me vine a meter!". Tuve una crisis de que no quería ir más a la universidad, algo fuerte, cuando pasé a segundo año. Me fui a Talca en las vacaciones y no quería volver a la universidad, decía: "No quiero, no quiero, no quiero ver a esa gente de nuevo, no quiero". Horrible. Tanto que mis papás me dijeron: "Si estás tan mal, no vayas", con todo el esfuerzo que yo sabía que significaba para ellos mantenerme en Santiago. (Profesional asalariada, 32 años, mujer, ingreso medio).

En términos prácticos, esto se traduce en tres tipos de reacciones que, según lo señalado por los entrevistados, son contingentes a elementos contextuales: la automarginación, el encubrimiento o la conformación de grupos referenciales similares. La primera se refiere a cuando los entrevistados perciben entre ellos y quienes comparten su posición de clase media una distancia insalvable, frente a la cual los sujetos prefieren mantener solo las relaciones estrictamente necesarias con quienes forman parte de este espacio, evitando la posibilidad de profundizar el vínculo.

En el segundo caso, los sujetos intentan encubrir aquello que los delata en su origen social, adoptando e imitando de manera sistemática las pautas de comportamiento del grupo de referencia y remitiendo el conocimiento de su origen social solo a relaciones de extrema confianza.

En el tercer caso, los individuos optan por establecer vínculos con sujetos que poseen una trayectoria similar, estableciendo una suerte de espacio protec-

\footnotetext{
11 Coloquial: de clase alta.

12 Coloquial: pasar inadvertido.
} 
tor frente a un contexto adverso. Estas tres actitudes no son excluyentes entre sí: en varios de los casos analizados, los entrevistados aluden a una o más actitudes de manera simultánea, otorgando un mayor o menor peso a cada una en determinados contextos y coyunturas de la propia biografía.

El segundo polo (orgullo de los orígenes) se configura a modo de respuesta frente a las dinámicas de discriminación a las que se ven expuestos los sujetos en el marco de su inserción paulatina en espacios de clase media. En este polo se reúnen las experiencias relacionadas con una integración del reconocimiento de la particularidad del lugar de origen y la revaloración del conocimiento que allí se obtuvo, lo que se traduce en tres reacciones: la reafirmación meritocrática, el resentimiento y la exposición voluntaria.

El primer elemento señala una actitud que relaciona directamente el logro de la posición del individuo con las características particulares identificadas en la idea de hazaña, por lo que se le concibe como una posición merecida en función de las capacidades desarrolladas y la actitud del sujeto:

Por ejemplo, [lugar de trabajo] tenía un par de gerentes que se creían la raja. Yo nunca les bajé la vista, ni los dejé mirarme en menos porque tenía más estudios, yo me sentía más preparado que los tipos. El tipo tenía la oficina llena de diplomas enmarcados de cursos de [lugar de trabajo]... Nunca en mi vida yo voy a enmarcar un curso de [lugar de trabajo]. Es demasiado mediocre. (Profesional en cargo directivo, 36 años, hombre, ingreso alto).

Esta reafirmación meritocrática también se manifiesta en relación con la clase trabajadora y los cercanos del hogar de origen, pues su propia experiencia en el sorteo de obstáculos genera un discurso fuertemente castigador hacia estos segmentos, en los que se identifica una actitud pasiva y apática en el mejoramiento de su calidad de vida.

La segunda reacción (el resentimiento) se relaciona directamente con las percepciones de desigualdad de los entrevistados, que identifican como injusto el excesivo costo tras el logro de la posición de clase media, en contraposición con quienes la obtienen sin mayor esfuerzo y que la comparten hoy día. Tras esta actitud hay una cierta amargura por lo sacrificado en el proceso, por la relectura del pasado de largo plazo, a la luz de la comparación con otros sujetos y con las facilidades que estos otros poseen en un contexto similar. Esta sensación de injusticia se manifiesta muchas veces en una actitud de rabia hacia quienes han tenido mayores facilidades en el logro de la posición de clase media:

Te da rabia porque uno, independiente de la situación que viva, encuentra que esa desigualdad no corresponde. Si fuera Opus Dei quizás creería que la movilidad 
social no existe y diría: "Yo soy pobre porque dios cree que yo soy pobre y bien". Pero, en general, no creo que sea así. Tú te encuentras en realidades súper contrapuestas, tanto en la universidad como en un lugar de trabajo, donde tú estás trabajando y eres el vendedor, y va un cliente que tiene mucha plata, y te compra miles de cosas y te trata pésimo y tú estás diciendo ahí: "Sí, sî" y tienes que agachar la cabeza porque él es el que tiene la plata para hacerlo. Siendo que tú no pediste estar donde estás y él no pidió estar donde está. La diferencia es que él si quiere está ahí, si quiere está allá o si quiere está en otro lado. Él hace lo que quiere y uno no. (Profesional asalariado, 33 años, hombre, ingreso medio).

Mi típica frase es "malditos millonarios, malditos privilegiados". Me da mucha rabia. Cuando hablaba, cuando hablo con mis ex compañeros de trabajo también me pasa. Hablábamos de cómo fue la infancia de cada uno, nada que ver, una distancia enorme, enorme. Años luz. Yo encargada de hacer las cosas de mi casa desde los nueve años, mientras mi mamá tejía en el taller, había que dividirse los quehaceres y estas chicas no: estaban leyendo, haciendo tareas o en clases de pintura y yo no tenía, no tuve esa posibilidad y claro que me da rabia. (Profesional asalariado, 32 años, mujer, ingreso medio).

La tercera actitud del polo del "orgullo del origen" (la exposición voluntaria) es una actitud complementaria a las otras dos arriba descritas y se relaciona con el establecimiento de diferencias con quienes comparten la posición de clase media, pero no el origen, mediante la exposición del origen y de los obstáculos en el logro de la posición. Con el establecimiento de esta diferencia, los entrevistados, por un lado, reafirman su propia creencia meritocrática, a partir del reconocimiento de su esfuerzo por parte de los pares y, por el otro, desarrollan una estrategia protectora frente a las dinámicas de discriminación que operan por la exposición del origen humilde de los entrevistados por parte de otros. Esa estrategia interviene como una respuesta anticipada a los cuestionamientos frente a la legitimidad de su condición de clase media:

Yo creo que hay que hablar de estas cosas en todas partes, hacerlo notar a los otros. A mí nada de esto me va a desmoralizar, porque yo sé que voy a conseguir lo que quiero, no sé si a toda costa, porque no va a ser así, pero yo sé que se puede, pero cuesta mucho más. A mí me costó estudiar también por lo mismo, porque no tenía los recursos, tenía que estar perdiendo tiempo, no estudiaba cien por ciento, porque tenía que trabajar, desde primero de la U. Desde antes incluso, desde el colegio, vendía papas fritas en el recreo, pero te cuesta mucho más todo si no eres hijo de alguien, porque yo me acuerdo, yo luchaba por mi beca de almuerzo y después veía en el patio al hijo de un diputado con la beca de 
almuerzo. Qué tiene que hacer él con beca de almuerzo. Entonces, yo no tenía problema en ir y decírselo: "Yo lo necesito y tú no". (Profesional asalariada, 33 años, mujer, ingreso medio).

\section{Los interlocutores}

Como se ha referido anteriormente, el discurso de la movilidad social está articulado en un permanente diálogo con varios interlocutores, frente a los cuales el sujeto elabora explicaciones, justificaciones o dirige palabras de agradecimiento. El primero de los interlocutores — quizás el más relevante- es la figura del impulsor, dentro del cual es posible distinguir dos versiones diferenciadas: el impulsor del hogar de origen y el impulsor externo. El primero se relaciona con una narrativa sobre la movilidad que, si bien involucra la totalidad del hogar, siempre identifica una figura clave que es la que imprime el deseo de "salir" en los entrevistados, la que motiva y acompaña el proceso. Este impulsor (mayoritariamente la madre, aunque no en forma exclusiva) es una figura ambivalente: por un lado, es un sujeto idealizado y depositario de agradecimiento, pero, por el otro, se le reconoce como un sujeto exigente, implacable y, en algunos casos, torpe por falta de herramientas, tanto durante el proceso como en la evaluación de resultados:

Mi papá no me dejaba juntarme con nadie. Yo tengo una hermana, que en ese tiempo vivíamos todos juntos, y mi hermana era mayor y tampoco ella podía jugar con ningún vecino, entonces, podíamos jugar no sé si media hora o una hora en la calle andando en bicicleta, y mi mamá nos miraba y de ahí entrábamos. Entonces, tanto tiempo que teníamos sin hacer nada porque no podíamos jugar con los otros niños, nosotros [...], bueno, leer, nos llevaba recortes, papeles de la imprenta, entonces jugábamos a la oficina, como a eso. Me incentivó harto la lectura y a él también le encantaba leer. Me llevaba El Mercurio los domingos, me compraba El Mercurio para que yo lo leyera, y era terrible y me hacía leer el "Artes y Letras". (Profesional asalariado, 33 años, mujer, ingreso medio).

Por otra parte, el impulsor externo es la figura que condensa a aquellas personas que, durante la trayectoria de movilidad social, se constituyen en un apoyo adicional y decisivo para el proceso de movilidad. Los entrevistados son conscientes de la falta de herramientas del impulsor del hogar de origen para elaborar estrategias en el logro de este objetivo, dadas las condiciones de marginalidad, pobreza y escaso nivel educacional que poseen. En este contexto, el impulsor externo adquiere sentido como el personaje que da forma y curso al 
impulso formado en el hogar de origen, marcando un punto de inflexión en la trayectoria biográfica narrada por el sujeto. En la mayoría de los casos, remite a la escuela pública, a partir de la figura de un profesor o director, generalmente ligada al ingreso a un liceo público de tradición, ${ }^{13}$ que deviene como el impulso definitivo para el acceso a la educación superior. En este punto, es relevante el rol de familiares lejanos o amigos de la familia con una posición social y nivel de ingreso mayor al del hogar de origen.

Este punto va en la línea de lo establecido por Espinoza (2006) cuando se refiere al rol del capital social en el proceso de movilidad social, en tanto que no solo otorga una cierta ampliación de las posibles trayectorias de vida imaginadas por el sujeto, sino que también establece los pasos concretos en el marco de la obtención de objetivos, instalando en el horizonte de lo posible cursos de vida antes inimaginables.

El segundo sujeto es el obstaculizador, este también se encuentra diferenciado en dos versiones en los discursos de los sujetos: el obstaculizador del hogar de origen y el obstaculizador externo. En la figura del obstaculizador, los entrevistados condensan una serie de individuos o circunstancias que dificultan el proceso de movilidad, desincentivándolo o directamente atentando contra él, de manera voluntaria o involuntaria. El obstaculizador del hogar de origen corresponde de forma predominante a los sujetos pertenecientes a la estructura familiar que no creen que la movilidad sea posible, desincentivando al sujeto o atentando contra sus esfuerzos.

En este punto es donde se sitúan dinámicas de maltrato, negligencia, alcoholismo, drogadicción o desinterés en fomentar en los nińos el proyecto de la movilidad por la vía de la educación. La figura del obstaculizador del hogar de origen, al igual que la figura del impulsor, tienen un carácter contradictorio: si bien se relata con amargura la falta de preocupación o las dinámicas de maltrato, también existe un cierto sentido de condescendencia que remite a las pocas posibilidades de ver otras alternativas de vida distintas a las del universo marginal. Si la relación que se establece con el impulsor es de retribución/deuda,

13 El modelo de escuela pública de tradición remite a los establecimientos gratuitos de secundaria que realizan un proceso de selección entre postulantes provenientes de diversos sectores del país, en función de un buen rendimiento académico. Estos liceos tienen una extensa tradición en la preparación de estudiantes para el ingreso al sistema universitario, e históricamente han sido cuna de numerosas figuras connotadas en el ámbito de la ciencia y la política. Dichos establecimientos son apuntados como espacios tradicionalmente vinculados a las clases medias. A la fecha, los estudiantes de estas instituciones son casi los únicos provenientes del sistema público que acceden a las universidades de prestigio nacional, y son también casi los únicos establecimientos públicos que figuran en los primeros puestos en lo que refiere al Sistema de Medición de Calidad de la Educación (Simce). Entre los más antiguos y conocidos, se hallan el Instituto Nacional, el Liceo no. 1 "Javiera Carrera", el Liceo Manuel Barros Borgońo, el Liceo Carmela Carvajal, por nombrar los más relevantes. 
la que se establece con este obstaculizador es la de demostración, a partir de la cual los sujetos buscan probar que, a pesar de la actitud de este, el sujeto fue capaz de lograr su objetivo:

Una vez me dijo mi papá: "Trabaja conmigo, métete en la construcción; te metes a un curso de albañil y trabajas conmigo; te meto en un trabajo tranquilo". Y yo: "Es que yo quiero ir a la universidad", y él: "No, seamos realistas, hijo". Después, cuando salí de la escuela, me dijo: "Yo ya cumplí, ahora si quieres algo más es cosa tuya", y yo le decía que quería entrar a la universidad y me decía: "Ya, pero hablemos en serio". "Papá, si yo quiero entrar a la universidad". Y después uno entró a la universidad, fue a la universidad y los papás están con el pecho inflado: "Mi hijo estudió en la universidad y en la Universidad de Chile". (Profesional asalariado, 33 años, hombre, ingreso medio).

Una relación similar es la que se establece con el obstaculizador externo, el que encarna a las personas fuera del hogar de origen que han dificultado o dificultan voluntaria o involuntariamente el proyecto del sujeto. Cuando la narrativa se remite al pasado de largo plazo, la figura remite a familiares lejanos, grupos de pares y, en algunos casos, a educadores o personas ligadas a la escuela pública que, a través de sus acciones, falta de motivación o apoyo, dificultan el proyecto del sujeto.

Cuando la narrativa se sitúa en el pasado reciente y presente, este obstaculizador se vincula a las barreras simbólicas y a las dinámicas de discriminación con las que debe lidiar el sujeto en lo cotidiano, encarnadas en un sujeto que en el marco de la competencia busca establecer una superioridad a partir del menoscabo de los individuos con un origen de clase trabajadora, ya sean profesores universitarios, compañeros o jefes en el espacio laboral:

La gente se fija, se fija en las camisas que usas, en la ropa que estás usando, si tu terno, si la camisa tiene tu nombre escrito o si usas lápiz Gross o Montblanc, se fijan en todo eso. Yo, por lo menos, yo lo he visto y se fijan en cosas que son más de embalaje, o sea, yo mido un metro y medio y soy morenito, eso también se fijan, incluso hay algunos que te lo tiran encima, te lo dicen, porque son cosas de origen. (Directivo profesional, 38 años, hombre, ingreso alto).

El tercer sujeto identificado en la narrativa son los pares significativos, los cuales corresponden a personas frente a las que se busca un reconocimiento y validación a lo largo del curso de vida. Estos pares significativos son diferenciados en tres tipos: los pares de origen, los pares de movilidad y los pares de la nueva posición. Los primeros son los individuos significativos de la posición 
de origen frente a los que los sujetos van desarrollando poco a poco una diferenciación y distancia, que se agranda a medida que el proceso de movilidad se vuelve exitoso: el contexto se va perdiendo y lo que constituía puntos de conexión se difumina paulatinamente. Pese a que en un primer momento los individuos tratan de seguir participando en los distintos espacios compartidos, prestando ayuda y apoyo a estos pares, en caso de ser necesario, en un momento posterior, el contacto se vuelve más esporádico y estos pares se transforman en pares "imaginados".

Por otro lado, los pares de movilidad son las personas con las que el sujeto genera una identificación, según aumente la distancia con los pares de origen. Cruzados por un mandato común, se constituyen en aquellos con los que se establece una mayor conexión en el contexto de extranjería en la que se ubican estos sujetos, en relación con otros en el marco de su proyecto de movilidad. Los pares de movilidad son con los que se establecen relaciones de complicidad en el marco de un contexto sentido como adverso por el sujeto: "Mi mundo personal principalmente está circunscrito a gente que es de esfuerzo, primera generación de profesionales en cada una de sus familias, la mayoría de mis amigos, o sea, mis amigos no son hijos de médicos ni de ejecutivos exitosos, mucho menos, son gente de esfuerzo no más." (Profesional en cargo directivo, 36 años, hombre, ingreso alto).

Parte del imperativo de no perder la conexión con los orígenes implica la conservación de este tipo de espacio: los entrevistados enfatizan que no han cambiado lo esencial de su identidad, recalcando que "mantienen los mismos amigos" y que "hacen las mismas cosas" que hacían antes con ellos, aun cuando su posición en términos estructurales se ha modificado notoriamente a lo largo del tiempo y aun cuando su grupo referencial no esté ya constituido por los pares de origen, sino por los pares de movilidad.

Los pares de la nueva posición son los que otorgan al sujeto la posición de interlocutor legítimo e integrante consolidado en la nueva posición. A lo largo de la experiencia de movilidad social, los sujetos deben adquirir conocimientos, modificar o desechar otros, y posicionarse como pares de otros sujetos de clase media que no comparten su origen, pero que les reconocen como interlocutores válidos.

Este proceso se produce a partir de un sinnúmero de vías, entre las más importantes se halla, en primer lugar, la experiencia educativa, que refiere no solo a la adquisición de conocimientos formales y credenciales educativas que le permiten introducirse en un mundo de clase media, sino también a toda la experiencia que rodea la adquisición de esos conocimientos y credenciales, que, en estos casos, está cruzada por la ruptura de la segregación espacial y el contacto sistemático con personas provenientes de distintas posiciones en tér- 
minos estructurales, con los cuales el individuo debe relacionarse, competir y validarse como un igual.

En segundo lugar, la inserción en el espacio laboral resulta también central, en tanto constituye un proceso en el que el sujeto debe adquirir experticia laboral mediante la acumulación de recursos a lo largo del tiempo y siendo validado entre los de su misma posición:

Yo tengo dos grupos de la vida que frecuento ahora: uno de gente que sí ha tenido un poco de apoyo económicamente en el ámbito familiar, normales. El otro es gente que conozco ahora por donde, por donde frecuento, y todos ellos son: "Hola cómo estás, yo soy apellido tanto y bla, bla, bla”, y la primera pregunta: “¿Y qué haces?, ¿¿dónde trabajas? y ¿dónde vas de vacaciones?, ¿qué auto tienes?”... puras tonteras. No me molesta, pero no soy parte de ellas. (Pequeña empresaria, 34 años, mujer, ingreso alto).

\section{Conclusiones}

En este artículo se analizaron discursos de los sujetos que se encuentran envueltos en un proceso de movilidad social ascendente, el cual adquiere una gran importancia en la construcción de sus relatos biográficos y en sus identidades como sujetos de "clases medias". A partir de los relatos de los actores analizados, mediante la metodología de la teoría fundamentada, se generaron tres categorías teóricas de mediano alcance, orientadas a la interpretación del significado que la experiencia de movilidad adquiere en términos subjetivos: movimiento ascendente, el mandato y la idea de fronteras/barreras. Dichas categorías de alcance intermedio constituyen un esfuerzo analítico en la comprensión de la dimensión cualitativa de estos procesos, hasta ahora relativamente ausente en los análisis del campo de los estudios de estratificación social en el caso chileno y latinoamericano.

La introducción de esta dimensión se relaciona con el hecho de que, si bien estamos hablando de procesos de largo plazo y de carácter macrosocial, estos modifican la experiencia cotidiana, la forma en que los sujetos estructuran su percepción del espacio social y, con ello, la relación entre grupos, Estado y la política. Resultan entonces fundamentales para comprender la sociedad contemporánea y la experiencia de vivir en Chile actual.

En este contexto, resulta particularmente interesante el rol que los interlocutores y el grupo familiar adquieren en este contexto. La noción de mandato, extraída del análisis del material cualitativo, habla de la dimensión más colectiva de la movilidad social y discute con las tesis de la prevalencia de una apuesta más individual en este proceso. 
Este fenómeno permite hipotetizar acerca de un desplazamiento de esta dimensión más colectiva, desde un espacio organizacional a grupos referenciales compuestos por la familia, pares de origen y otras redes. En ese sentido, esta transformación tendría impactos relevantes en el comportamiento de estos grupos a nivel político y a nivel de percepciones de la sociedad, entre otros aspectos. Queda abierta la posibilidad de probar esta y otras hipótesis en un futuro.

Este artículo buscó avanzar en una reflexión en esta dirección, rescatando el papel que las dinámicas de diferenciación y de recreación de fronteras simbólicas cumplen en la configuración de la experiencia de habitar en Chile contemporáneo. A la vez que se mostraron caminos o vías para posibles aplicaciones comparativas de estas categorías en el análisis cualitativo de otros casos similares en la región, previo ajuste contextual de las categorías.

La importancia del desarrollo de esta mirada comparativa, en un contexto de procesos similares de países de la región es invaluable, sobre todo si se considera que Chile es uno de los países pioneros en ciertas transformaciones económicas que han modificado los patrones de movilidad. Queda como tarea pendiente para futuras investigaciones en este campo.

\section{Referencias}

Arellano, R. (2010). Valores e ideología: el comportamiento político y económico de las nuevas clases medias en América Latina. En Bárcena, A. \& Serra, N. (Eds.). Clases medias y desarrollo en América Latina. Barcelona: CEPAL/CIDOB.

Ariztía, T. (2009). Arreglando la casa propia: La cultura material de la movilidad social. En Pérez, F. \& Tironi, M.S.C.L. Espacios, prácticas y cultura urbana (pp. 222-240). Santiago de Chile: ARQ.

Balbontín, I. (2007a). Actuales orientaciones políticas de las capas medias en Chile. Ciencias Sociales Online, 4(3).

Balbontín, I. (2007b, 14 de mayo). Concertación vs. alianza por la conquista de las clases medias. Informe No 605. Asuntos Públicos.

Barozet, E. \& Espinoza, V. (2008). ¿Quiénes pertenecen a la clase media en Chile? Una aproximación metodológica? Revista Ecuador Debate, 74, 103-121.

Bengoa, J., Márquez, F. \& Aravena, S. (1999). La desigualdad. Santiago de Chile: Ediciones Sur. 
Bertaux, D. (1997). Pathways to Social Class: A qualitative Approach to Social Mobility. Oxford: Clarendon Press.

Bourdieu, P. (2000). ¿Cómo se hace una clase social? En Poder, derecho y clases sociales. Bilbao: Desclée de Brouwer.

Bourdieu, P. (1999). La miseria del mundo. Buenos Aires: Fondo de Cultura Económica.

Bourdieu, P. (1979). La distinción. Criterio y bases sociales del gusto (María del Carmen Ruiz de Elvira, Trad.). Madrid: Taurus.

Bourdieu, P. \& Passeron, J. C. (2003). Los herederos. Buenos Aires: Siglo XxI.

Cabalin, C. (2012). Identidad cultural y ciudadanía en los sectores pobres de Santiago de Chile. Perfiles Latinoamericanos, 20(40), 123-142.

Campero, G. (1987). Entre la sobrevivencia y la acción politica. Las organizaciones de pobladores en Santiago. Santiago de Chile: Ediciones ILET.

Castillo, M. (2009). Identidades politicas. Trayectorias y cambios en el caso chileno. México: Flacso México.

Cerda, C. (1998). Historia y desarrollo de la clase media en Chile. Santiago de Chile: Ediciones Universidad Tecnológica Metropolitana.

Espinoza, V. (2006). La movilidad ocupacional en el Cono Sur. Oportunidades y desigualdad social. Revista de Sociología, 20, 131-146.

Glaser, B. G. (1998). Doing Grounded Theory: Issues and Discussions. Mill Valley, Cal.: Sociology Press.

Grynspan, R. \& Paramio, L. (Coords.). (2012). Clases medias en sociedades desiguales. Pensamiento Iberoamericano, 10(1). Recuperado de http://www.fundacioncarolina.es/wpcontent/ uploads/2014/07/PensamientoIbero10.pdf

Hall, S. (2000). Who Needs Identity. En Du Gay, P., Evans, J. \& Redman, P. (Eds.). Identity. A Reader. Londres: Sage.

León, A. \& Martínez, J. (2001). La estratificación social chilena hacia fines del siglo XX. Santiago de Chile: onu/CEPAL (Serie Políticas Sociales, 52).

Londoño, J. (2011). Movilidad social, preferencias redistributivas y felicidad en Colombia. Revista Desarrollo y Sociedad, 68, 172-212. 
López-Calva, L. F. \& Juárez Ortiz, E. (2012). Pensamiento Iberoamericano, 10, 281-307.

López-Calva, L. F., Rigolini, J. \& Torche, F. (2012). Is There Such Thing as Middle Class Values? Class Differences, Values and Political Orientations in Latin America. Discussion Paper series, Forschungsinstitut zur Zukunft der Arbeit, 6292. Recuperado de http://hdl.handle .net/10419/58515

Martínez, J. \& Tironi, E. (1985). Las clases sociales en Chile. Cambio y estratificación, 1970-1980. Santiago de Chile: Ediciones Sur.

Martínez, J. \& Tironi, E. (1982). Materiales para el estudio de las clases medias en la sociedad chilena 1960-1980. Documento de Trabajo, (21). Santiago de Chile: Flacso Chile.

Méndez, M. L. (2009a). Space Matters: Authenticity of Place as a Vital of Middle Class Urban Politics. Draft for Latin American Studies Association Congress.

Méndez, M. L. (2009b). Clases medias en Chile: transformaciones, sentido de pertenencia y tensiones entre proyectos de movilidad. En Franco, R., Hopenhayn, M. \& León, A. (Eds.). Clases medias en América Latina. Retrospectiva y cambios recientes. México: CEPAL/Siglo XXI Editores.

Méndez, M. L. \& Barozet, E. (2012). Lo auténtico también es público. Comprensión de lo público desde las clases medias en Chile. Revista Polis, 31.

Méndez, M. L. \& Gayo, M. (2007). El perfil de un debate: movilidad y meritocracia. Contribución al estudio de las sociedades latinoamericanas. En Franco, R., León, A. \& Atria, R. (Eds.). Estratificación y movilidad social en América Latina. Santiago, Chile: CEPAL/LOM Ediciones.

Portes, A. \& Hoffman, N. (2003). Las estructuras de clase en América Latina: composición y cambios durante la época neoliberal. Santiago de Chile: cepal (Serie Políticas Sociales, 68).

Posner, P. (1999). Neoliberalism and Democracy: The State and Popular Participation in PostAuthoritarian Chile. Chapel Hill: University of North Carolina at Chapel Hill.

Sánchez Salcedo, J. (2007). Gente bien, gente decente: Fronteras simbólicas, procesos de identificación y diferenciación de profesionales pertenecientes a las clases medias en Cali. Revista Cientifica Guillermo de Ockham, 5(1), 85-100.

Silva, B. (2005). La clase media después de las transformaciones estructurales: una aproximación cualitativa a través del análisis de clase. Tesis para obtener el grado de socióloga. Santiago de Chile: Universidad de Chile. 
Solimano, A. (2005). Hacia nuevas políticas sociales en América Latina: crecimiento, clases medias y derechos sociales. Revista de la CEPAL, (87), 1-208.

Stillerman, J. (2010a). The Contested Spaces of Chile's Middle Classes. Political Power and Social Theory, 21, 185-214..

Stillerman, J. (2010b). The Search for Middle Class Housing in Santiago: Mapping the Interactions between Taste, Family Resources, and Housing Markets. Toronto. Ponencia presentada en el Congreso de la Asociación de Estudios Latinoamericanos (LASA).

Svampa, M. \& Bombal, I. (2001). Movilidad social ascendente y descendente en las clases medias argentinas: un estudio comparativo. Documento de Trabajo. Siempro/Unesco.

Torche, F. (2005). Desigual pero fluido: el patrón chileno de movilidad en perspectiva comparada. Santiago de Chile: Expansiva (Serie en Foco, 57).

Torche, F. \& López-Calva, F. (2012). Stability and Vulnerability of the Latin American Middle Class. Oxford Development Studies.

Torche, F. \& Wormald, G. (2007). Chile, entre la adscripción y el logro. En Estratificación y movilidad social en América Latina. Transformaciones estructurales de un cuarto de siglo. Santiago de Chile: Lom Ediciones.

Visacovsky, S. (2012). Experiencias de descenso social: percepción de fronteras sociales e identidad de clase media en la Argentina post-crisis. Pensamiento Iberoamericano, 10, 133-168.

Recibido el 16 de mayo de 2014. Aceptado el 5 de junio de 2015. 